(Aus dem histologischen Institut und der Frauenklinik der Universität München.)

\title{
Beitrag zur Kenntniss der decidualen Bildungen in den Ovarien bei intrauteriner Graviditäit ${ }^{1}$ ).
}

\author{
Von \\ Priv.-Doc. Dr. Karl Hörmann, \\ I. Assistent der Klinik. \\ (Hierzu Tafel XI.)
}

Die histologischen Untersuchungen der letzten Jahre haben ergeben, dass sich beim Menschen während der Schwangerschaft nicht nur in der Schleimhaut des Uterus eine deciduale Umwandlung des Stromas findet, sondern dass in einer sehr grossen Anzahl von Fällen, ja fast regelmässig auch an mannigfachen anderen Stellen des Genitaltractus und seiner Nachbarschaft ganz unabhängig von der Insertionsstelle des Eies - deciduaähnliches Gewebe auftritt. Man pflegt von einer decidualen Reaktion der Gewebe auf die Eiansiedelung zu sprechen. Walker, $\mathrm{Z}_{\text {weifel }}$ u. a. fanden zunächst grosszelliges, deciduaähnliches Gewebe bei Extrauteringravidität in dem Beckenbauchfell, Pels-Leusden und Schmorl konstatirten analoge Befunde dann auch als eine sehr häufige Begleiterscheinung der normalen Schwangerschaft, und zwar am deutlichsten in Form von grauweisslichen Knötchen oder stärker injicirten Platten in der Serosa am Boden des Douglasschen Raumes, an der hinteren Wand des Uterus und der vorderen Wand des Mastdarmes. Schmorl hat diese deciduaähnlichen Haufen zuerst auch in den Ovarien gravider Frauen nachgewiesen,

1) Nach einem in der Münchener Gesellschaft für Morphologie und Physiologie gehaltenen Vortrag. 

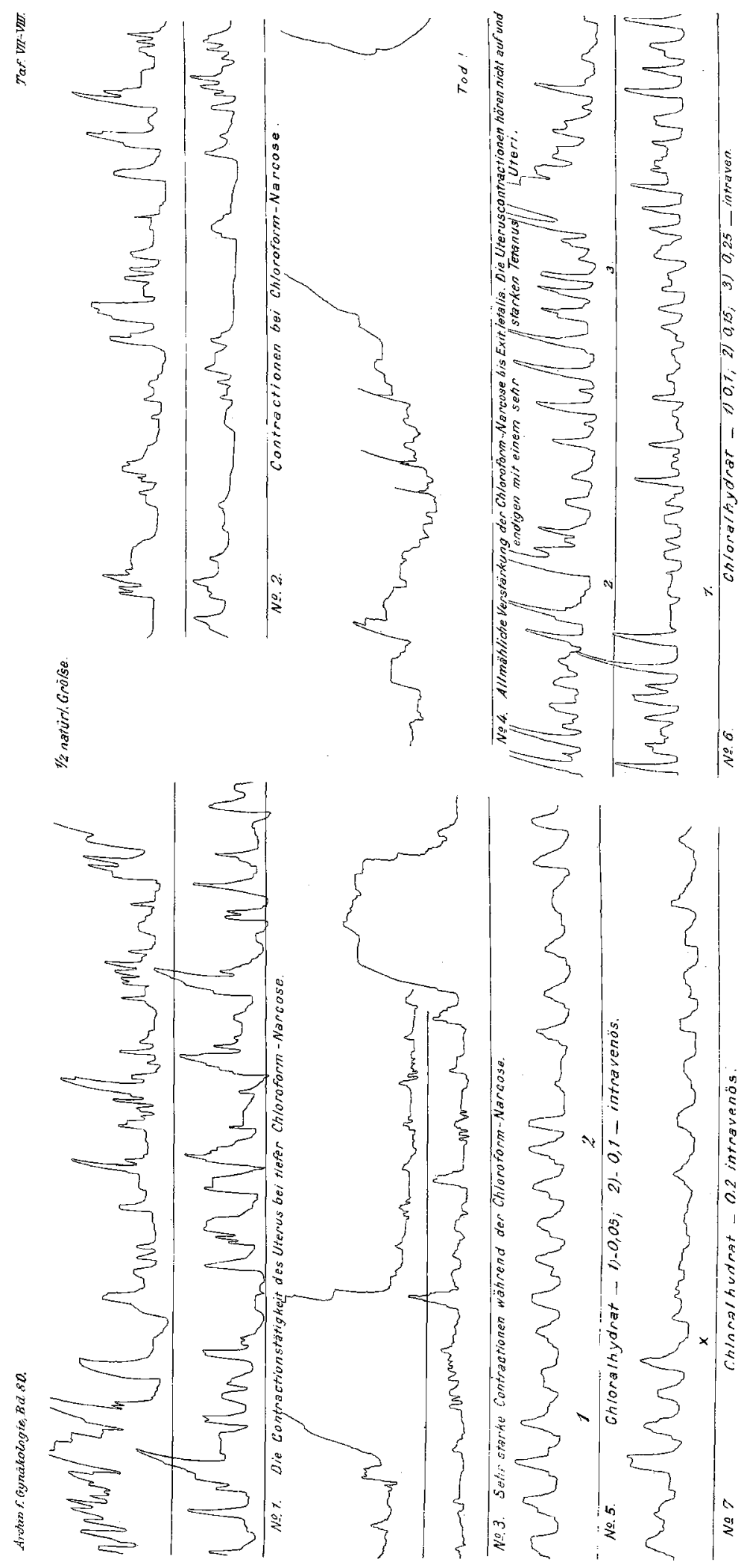
298 Hörmann, Deciduale Bildungen i. d. Ovarien b. intrauteriner Gravidität.

und seine Angaben haben seither mehrfache Bestätigung, vor allem durch Kinoshita, Schnell, Rabl und Lindenthal, gefunden. Die fraglichen Zellgruppen liegen meist dicht unter dem Keimepithel, aber auch tiefer, und werden vom 5. Monat ab als Knötchen von blassgrauer Farbe häufig schon makroskopisch sichtbar.

Wie häufig das Auftreten jener eigenthümlichen Zellherde in den Ovarien Gravider ist, geht aus den Angaben von Lindenthal, der sie unter 34 Fällen 25 mal, und Schnell, der sie unter 20 Fällen 17 mal fand, hervor. Charakteristisch für die in Frage stehende Gewebsveränderung ist ihr Entstehen während der Schwangerschaft, ihr Verschwinden am Ende derselben und ihre grosse morphologische Aehnlichkeit mit dem Deciduagewebe der Uterusschleimhaut bei intrauteriner Gravidität. Lindenthal konnte Ovarien aus den verschiedenen Monaten der Schwangerschaft untersuchen und sowohl die Entstehung wie die Rückbildung dieser Formationen verfolgen; er fand sie vom Ende des 3. Lunarmonats an; im 9. und 10. Monate aber erreichen dieselben erst ihre grösste Ausbildung; unter 16 untersuchten Fällen fehlten sie hier nur 3 mal. Beginnt schon im 7. Monat eine Art hyaliner Degeneration oder Sklerose an einzelnen Zellgruppen, so werden am Ende der Gravidität die. Rüekbildungserscheinungen sehr ausgedehnt beobachtet, in Form von Vacuolisirung des Protoplasmas, Veränderung der Kernstructuren und hyaliner Umwandlung ganzer Zellgruppen. Im Puerperium scheint dann die Degeneration der deciduaähnlichen Herde sehr rasch fortzuschreiten, sodass nur noch in den ersten Tagen des Wochenbettes unveränderte Zellgruppen gefunden wurden; schliesslich bleiben hyaline oder kernarme Stellen zurück.

Die histologische Struktur der in voller Ausbildung befindlichen grosszelligen Herde wird von den oben erwähnten Autoren, soweit sie sich eingehender mit diesen Bildungen beschäftigt haben, nicht ganz übereinstimmend gesehildert. Dieser Umstand, sowie der Wunsch, eventuell sichere histologische Anhaltspunkte für die Identität der grossen Zellen mit den Elementen der Decidua uterina aufzufinden, veranlassten mich, diesen eigenartigen Bildungen gelegentlich der Untersuchung einer Anzahl von Ovarien gravider Frauen zu anderen Zwecken ein besonderes Interesse zuzuwenden. Im Folgenden sei es mir gestattet, meine Resultate mitzutheilen. Alle untersuchten Objecte waren ganz frisch gewonnen und in 5 proc. Formollösung fixirt worden. Die Färbung der ange- 
Hörmann, Deciduale Bildungen i. d. Ovarien b. intraciteriner Gravidität. 299

fertigten Paraffinschnitte gesehah zum Theil nach den gewöhnlichen Methoden, z. B. Hämatoxylin-Eosin, zum Theil aber wurden spezielle Färbemethoden angewendet, die allein gewisse Details in genügender Deutlichkeit erkennen liessen. Sie sind bei Beschreibung der entsprechenden Befunde aufgeführt.

Wie die Betrachtung eines Uebersichtsbildes (Taf. XI, Fig. 1) aus dem Ovarium einer Gravida vom 10. Monat ${ }^{1}$ ) zeigt, erinnern in der That die meisten der Zellen in den Knoten nach Form, Grösse, Anordnung, Beschaffenheit des Kernes und Verhältniss desselben zum Zellenleib ohne weiteres an die so charakteristischen Zellen der Decidua uterina; sie sind sehr vielgestaltig, unregelmässig rundlich, oval, kurzspindelig, kolbig, birnenförmig und auffallend gross. Die Zellen liegen in vielen Knoten so dicht aneinander, dass man bei oberflächlicher Betrachtung, wie Schmorl hervorhebt, den Eindruck gewinnt, als ob man es mit geschichtetem Plattenepithel zu thun hätte. Sobald man aber eine specifische Bindegewebsfärbung vornimmt, treten zwischen den Zellen aufs deutlichste sehr feinfaserige Intercellularbildungen hervor. Schmorl bediente sich zum Nachweis derselben der van Gieson'schen Färbemethode. Das in der 2. Figur abgebildete Präparat ist nach Maresch behandelt, der auf eine für Bindegewebsfasern sehr brauchbare Modification der Bielschowsky'schen Nervenfibrillen-Färbungsmethode aufmerksam gemacht hat. (Da ich beabsichtige, demnächst des eingehenderen über die mit dieser Methode erzielten Befunde an den weiblichen Genitalorganen zu berichten, möchte ich vorläufig auf eine genauere Besprechung derselben verzichten. Im Vergleich mit der van Gieson'schen Methode ist dieses Verfahren viel exacter und lässt auch die feinsten Fäserchen infolge der intensiven Farbe deutlich und scharf hervortreten.) Ueberall sieht man (Taf. XI, Fig. 2) aufs schärfste zwischen den grossen. Zellen ein Netzwerk von feinsten Fasern, so dass die Zellen von diesem Filzwerk umsponnen werden. Ich kann also gegenüber Lindenthal, der eine solche different gefärbte Zwischensubstanz niemals nachweisen konnte, die Angaben von Schmorl und Kinoshita durch unzweideutige Präparate bestätigen. Uebrigens kann man die Kerne der zu diesen Fasern gehörigen Fibroblasten auch schon an mit Hämatoxylin-

1) Dieselbe erlag in kürzester Zeit einer sub partu eingetretenen ausgedehnten Uterusruptur. 
है

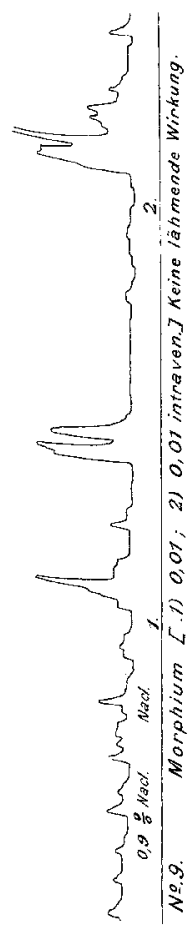

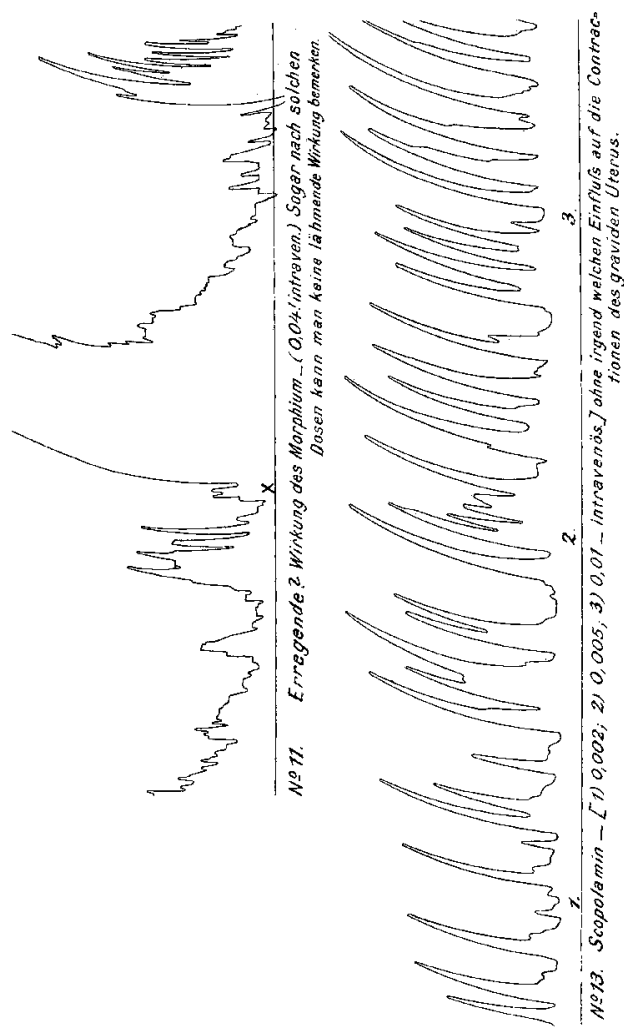

$\frac{1}{3}$
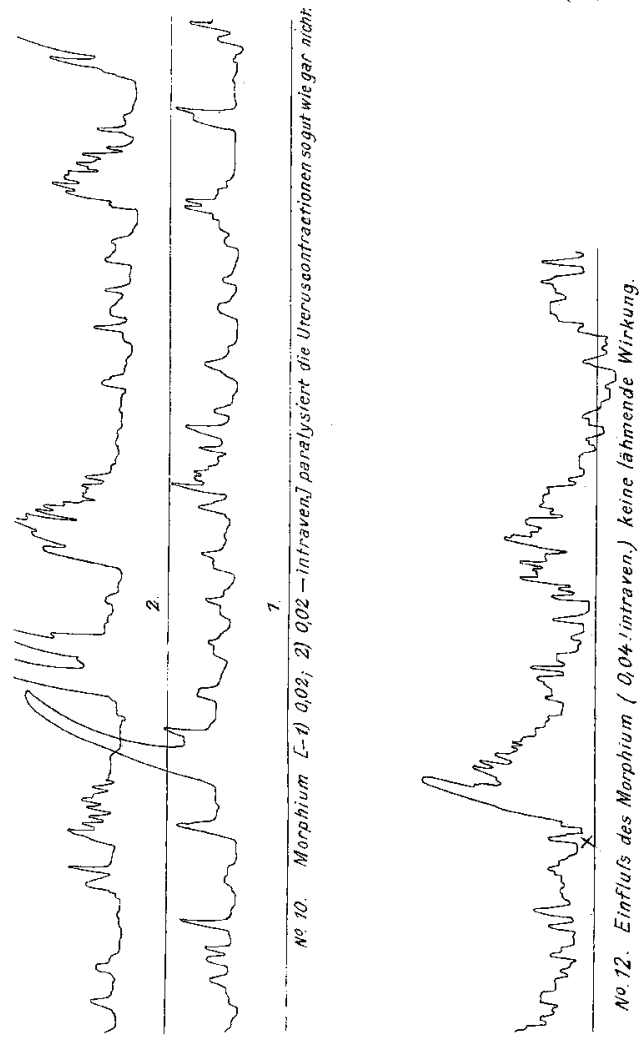
300 Hörmann, Deciduale Bildungen i. d. Ovarien b. intrauteriner Gravidität.

Eosin gefärbten Präparaten durch ihre Kleinheit zwischen den grossen decidualen Zellen erkennen. Die Figur 3 illustrirt dieses Verhältniss deutlich; einige der hier mit eingezeichneten, besonders dunkel tingirten, rundlichen, kleinen Zellen mögen auch Lymphocyten sind.

Die in den gebräuchlichen anatomischen und geburtshilflichen Lehr- und Handbüchern von der Decidua uterina gegebenen Beschreibungen liessen es mir zweifelhaft erseheinen, ob auch zwischen den Deciduazellen (vor allem in der compacten Schicht) eine ähnliche, feinfaserige Intercellularsubstanz bereits bisher angenommen bezw. dargestellt worden sei. Mit Ausnahme der etwas unbestimmten Angabe Pfannenstiel's in v. Winckel's Handbuch der Geburtshilfe (Bd. I/1, S. 212), dass "die Deciduazellen miteinander sowie mit dem intercellulären Fasergerüst vielfach durch dünnere und stärkere Fortsätze verbunden seien", fand jch nirgends etwas davon erwähnt oder abgebildet. ${ }^{1}$ ) Ich habe darum auch versucht, zwischen den Zellen der Decidua compacta dasselbe Faserwerk wie in den ovariellen Haufen nachzuweisen, und es gelang mir dies ebenfalls mittels der oben erwähnten Methode nach Maresch an zwei ganz frisch gewonnenen, in 5 proc. Formollösung fixirten Deciduae; doch sind die Fasern hier etwas weniger zahlreich ${ }^{2}$. Die gewöhnliche Darstellung, als ob die Deciduazellen der Mucosa uteri ohne faserige Intercellularsubstanz dicht aneinander lägen, ist also nicht richtig, und die Analogie zwischen den "decidualen" Haufen im Ovarium und der uterinen Decidua (compacta) wird durch den mitgetheilten Befund eines intercellulären Faserwerkes nicht beeinträchtigt; im Gegentheil gefestigt.

Betrachten wir nun die grossen, auffallenden Zellen, welche die ovariellen Haufen in der Hauptsache zusammensetzen, etwas näher, so zeigen sie, wie die uterinen Deciduazellen einen grossen, gut färbbaren Kern. Es kommen auch zwei- und dreikernige Zellen und gewöhnliche Riesenzellen, freilich relativ selten, in den Haufen vor. Mitosen dagegen sind nur ganz ausnahmsweise zu sehen, wiewohl die Objecte ganz frisch und lebenswarm fixirt wurden.

1) Anmerk. bei der Correktur: Nachträglich fand ich noch in dem Grundriss von $\mathrm{Bumm}$ eine diesbozügliche positive Angabe (S. 65).

2) Die zu den Fasern gehörigen Bindegewebszellen sind ebenfalls deutlich za sehen und durch den bedeutenden Grössenunterschied und die Beschaffenheit des Kernes leicht von den Deciduazellen zu unterscheiden. 

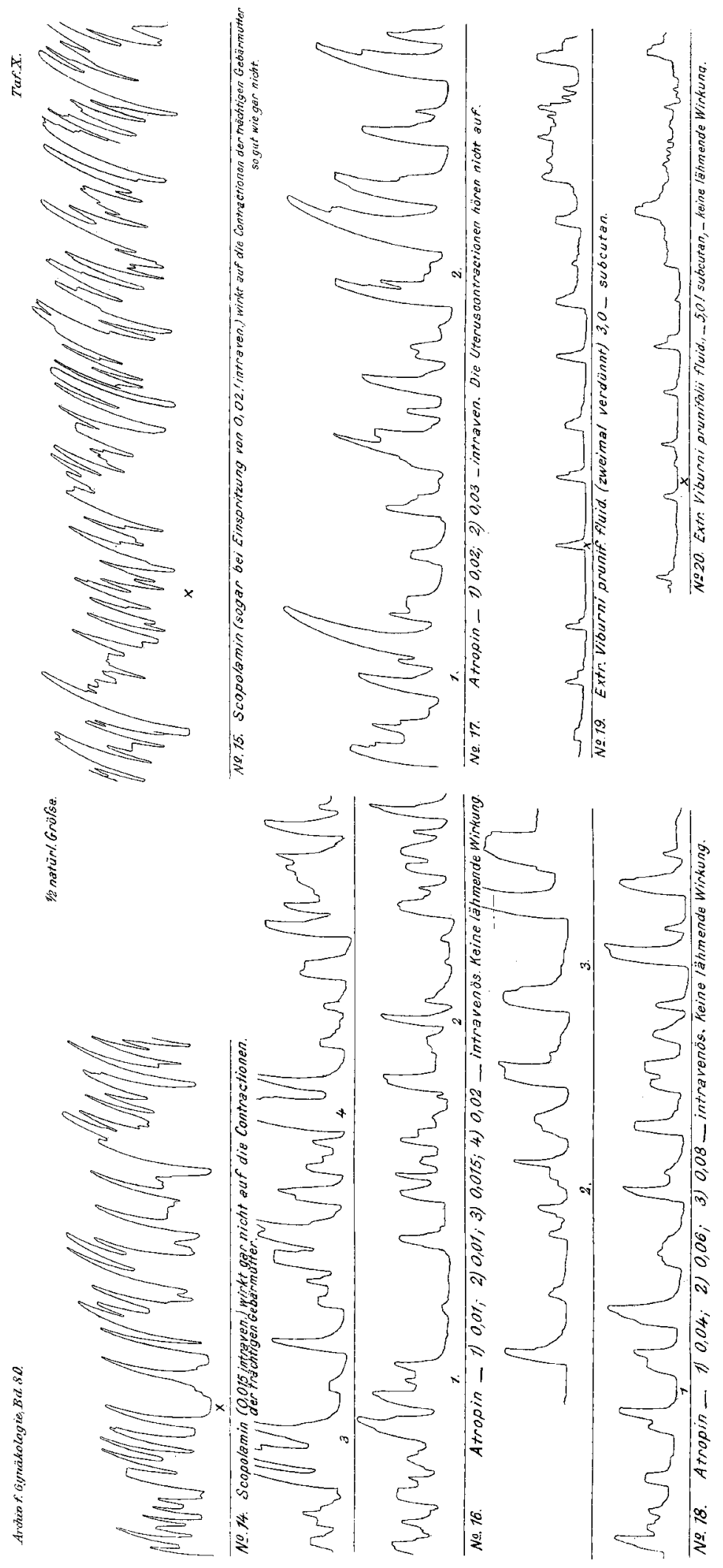
Hörmann, Deciduale Bildungen i. d. Ovarien b. intrauteriner Gravidität. 301

Der Zellenleib ist häufig lıomogen und mit Eosin gleichmässig färbbar, nicht selten aber nimmt das Protoplasma, wie es auch Schmorl in älteren Haufen beobachtete, eine netzförmige, schaumige Structur an, die wohl auf degenerative Processe zurückzuführen ist. Insbesondere ist des Ferneren zu erwähnen, dass in den Zellhaufen ausser diesen den "Deciduazellen" im engeren Sinne so ähnlichen Elementen auch syncytiale Zellen in variabler Zahl vorkommen. Der syncytiale Charakter der Zellen ist beispielsweise deutlich in dem auf der 4. Figur (Tafel XI) abgebildeten Präparat: durch Ausläufer stehen die einzelnen Zellen unter einander in directer Verbindung, sie bilden echte Syncytien; ihre Tinktionsfühigkeit mit bestimmten Farbstoffen ist eine auffallend starke. Vielfach bemerkt man Einschnürungen an ihrem Zellkörper; man sieht z. B. sanduhrförmige Zellen mit je einem Kern diesseits und jenseits der Einschnürung; solche Bilder lassen vielleicht an directe Theilungsvorgänge denken.

Das Vorkommen von Syneytien bezw. syncytialen Zellen in den ovariellen Haufen wurde bisher von keinem Beobachter ausdrücklich betont; da auch in der uterinen Decidua solche syncytiale Bildungen, wie gerade neuere Untersuchungen bestätigen, nichts Seltenes sind, so kann uns dieser Befund bei der angenommenen Analogie nicht überraschen. Ohne hier auf den alten Streit über die Herkunft der syncytialen Zellen in der Decidua basalis eingehen zu wollen, möchte ich nur darauf hinweisen, dass der Befund von Syncytien in den ovariellen Herden aufs Neue zeigt, wie die verschiedensten Gewebsarten während der Gravidität zur syncytialen Umwandlung neigen. Es ist also nicht einwandsfrei, wenn allein aus der morphologischen Aehnlichkeit der syncytialen Zellen in der Decidua serotina mit dem syneytialen Zottenüberzug auf ihre Herkunft von dem letzteren geschlossen wird; vielmehr lässt sich ihre Entstehung aus den Gewebselementen der Decidua selbst, also aus maternem Gewebe, ebenso wie in jenen ovariellen Haufen, sehr wohl annehmen.

Beim weiteren genauen Durchsuchen jener grossen "decidualen" Zellen in den ovariellen Herden auf besondere, eventuell charakteristische Merkmale hat sich fast constant ein auffallender Befund an ihnen ergeben; in beinahe allen liessen sich nämlich Centrosomen mit grösster Leichtigkeit und Deutlichkeit darstellen. Dieser Befund war um so beachtenswerther, als der Nachweis dieser Gebilde in ruhenden Zellen des menschlichen Organismus in dieser 
302 Hörmann, Deciduale Bildungen i. d. Ovarien b. intrauteriner Gravidität.

Klarheit im Allgemeinen durchaus nicht leicht ist. In den beiden ersten unter Fig. 5 Taf. XI abgebildeten Zellen sieht man z. B. in einiger Entfernung von dem ruhenden Kern je ein tiefschwarz gefärbtes Centrosom in einem heller (in den zugehörigen Präparaten röthlich) gefärbten Hof, der Centrosphäre; an manchen besonders schönen Präparaten war auch eine radiäre Strahlung um die Centrosphäre wahrzunehmen. Sehr häufig war der Befund von zwei Centrosomen neben einem ruhenden Kern, und zwar manchmal ganz nahe beisammen, manchmal aber auffallend weit von einander entfernt (cfr. die übrigen Zellen der Fig. 5, Taf. XI). In ersterem Falle haben beide Centrosomen des Oefteren eine gemeinsame Sphäre (Zelle 3) mit sanduhrförmiger Einschnürung, woraus auf eine vor sich gehende Theilung des Centrosoma mit Betheiligung der Sphäre geschlossen werden könnte; im letzteren Falle hat jedes Centrosoma seine eigene Sphäre (Zelle 4, 5 u. 6 der Fig. 5 Taf. XI). ${ }^{1}$ ) Bemerkenswerth ist die Lage der Centrosomen, insofern sie auffallend weit von dem stets in Ruhe befindlichen Kern entfernt sind, sowie ihre bedeutende Grösse. In Folge der letzteren Eigenschaft ist auf ihre Anwesenheit schon bei gewöhnlicher Färbung der Präparate, z. B. mit Hämatoxylin-Eosin, zu schliessen; so deutlich wie in den auf Fig. 5 Taf. XI abgebildeten Zellen werden sie allerdings erst bei Anwendung specieller Methoden, hier der M. Heidenhain'schen Eisen-Hämatoxylinfärbung. ${ }^{2}$ ) Auch mit anderen Färbemethoden, z. B. nach Mallory, BiondiEhrlich-Heidenhain lassen sich die Centrosomen auffallend leicht darstellen.

Man könnte zunächst geneigt sein, aus der Anwesenheit so zahlreicher Centrosomen auf eine ausgedehnte productive Thätigkeit in jenen Zellhaufen zu schliessen; dem ist aber in den von mir untersuchten Stadien (gegen Ende der Gravidität) nicht so. Im Gegentheil, in Uebereinstimmung mit früheren Beobachtern (Schmorl, Lindenthal u. A.) konnte auch ich an den in Frage stehenden Zellen auffallend häufig degenerative Vorgänge con-

1) Statt eines Centrosoms sieht man manchmal einen Haufen tiefschwarzer Pünktchen an der entsprechenden Stelle; vielleicht deutet djes auf einen Zerfall des Centrosoms hin.

2) Technisch ist vielleicht zu bemerken, dass beim Ausziehen der Präparate mit Eisenammoniumsulfatlösung die Haufen allein zuerst hell (,fertig") werden, während alle übrigen Stellen des Präparates noch pechschwarz sind. Nähere Beschreibung der Methode siehe z. B. Böhm u. Oppel, Taschenb. d. mikroskopischen Technik, 1904. No. 199. 
Hörmann, Deciduale Bildungen i. d. Ovarien b. intrauteriner Gravidität. 303

statiren: Die Kernmembranen sind oft geschrumpft, das Chromatin zusammengeballt, klecksig und klumpig, Pyknosen und Karyolysen sind häufige Befunde. Auch das Protoplasma des Zellenleibes erscheint, wie schon oben erwähnt, des Oefteren schaumig, netzförmig, vacuolisirt. So ziemlich alle Kerne sind im ruhenden $\mathrm{Zu}$ stande, sehr selten wurden Mitosen gesehen. Gleichwohl enthalten auch alle diese mit den Zeichen der Degeneration versehene Zellen Centrosomen. Somit lässt sich von diesen Gebilden in unserew Falle bei dem Fehlen aller productiven Vorgänge in den Kernen nur sagen, dass sie sich auffallend lange und gut sogar in untergehenden Zellen erhalten können. Der häufige Befund von zwei Centrosomen mit einer sanduhrförmig eingeschnürten Sphäre könnte vielleicht so gedeutet werden, dass sie vor ihrem Untergange sogar einen Anlauf zur Zweitheilung machen ${ }^{1}$ ); ihre auffallende Grösse könnte mit einer Quellung vor ihrem Untergange zusammenhängen.

Alle oben beschriebenen und abgebildeten Befunde wurden zunächst an ein und demselben Objecte, dem Ovarium einer sub partu an Uterusruptur verstorbenen, im 10. Monate graviden Frau, erhoben. Es lag nun natürlich nahe, die Constanz dieser histologischen Structurveriältnisse an einigen anderen Objeeten nachzuprüfen; ohne besondere Auswahl wurden hierzu die Ovarien einer im 6. Schwangerschaftsmonat an Eklampsie und einer am Ende der Gravidität an Uterusruptur verstorbenen Frau verwendet. Dieselben waren ebenso frisch in 5 proc. Formollösung fixirt worden wie das zuerst untersuchte Ovarium. Das Resultat deckte sich in jeder Beziehung mit den Befunden am ersten Ovarium, so dass ich von jeder weiteren Beschreibung absehen kann. Da insbesondere auch die Centrosomen in den deciduaähnlichen Zellen ebenso constant deutlich und leicht mit den verschiedenen oben erwähnten Färbemethoden darzustellen waren, so hat dieser Umstand auch ein gewisses technisches Interesse, insofern die decidualen Herde im Ovarium ein brauchbares Object zur demonstrativen Darstellung jener Gebilde zu sein scheinen.

Um ausser den bisher erörterten morphologischen Analogien zwischen den Deciduazellen des Uterus und jenen grossen Zellen in den Ovarien noch weitere Stützpunkte für ihre eventuelle Identität zu gewinnen, wurden auch die uterinen Deciduazellen

1) Damit wäre auch hier ihre Unabhängigkeit von der Kernsubstanz documentirt. 
304 Hörmann, Deciduale Bildungen i. d. Ovarien b. intrauteriner Gravidität.

auf Centrosomen untersucht. Da mir zunächst nur einige ältere, offenbar nicht ganz geeignet (in Alkohol) fixirte Deciduae zur Verfügung standen, misslang der Nachweis der Centrosomen für's Erste. Im letzten Augenblick vor Abschluss dieser Arbeit spielte mir aber der Zufall noch zwei ganz frische Deciduen aus der 7.-8. Schwangerschaftswoche in die Hände, die ich völlig frisch in 5 proc. Formollösung fixiren konnte. An diesen vorzüglich conservirten Objecten waren sowohl $\mathrm{zw}$ wschen den Deciduazellen die früher erwähnten feinen Bindegewebsfasern wie innerhalb der Zellen die Centrosomen ebenso constant wie in den ovariellen Haufenzellen nachzoweisen. Häufig fiel hier eine eigenthümliche Form der Centrosomen in die Augen, indem sie das Aussehen von Hufeisen oder Stäbchen hatten; im Uebrigen waren solche Formen auch in den "ovariellen Deciduazellen" bei nochmaliger Controle der Präparate aufzufinden, wenn auch hier die kreisrunden bezw. kugeligen Formen in der Ueberzahl waren. Wenn man gleichmässig vorbehandelte und gefärbte Schnitte aus den zuletzt untersuchten frischen Deciduen und den ovariellen Haufen unter dem Mikroskope vergleicht, so wird es auch dem geübten Beobachter schwer werden, Unterschiede in den histologischen Structurverhältnissen aufzufinden; die Uebereinstimmung ist auch in den feineren. Einzelheiten eine ganz auffallende. Ich kann also der Ansicht der früheren Untersucher, welche jene eigenthümlichen grosszelligen Herde im Ovarium mit der uterinen Deciduabildung in Parallele setzten, vollkommen beistimmen und glaube folgende gemeinsame Befunde als weitere Stützpunkte für die Identität der beiden Bildungen erbracht zu haben:

1. den Nachweis eines feinen intercellulären Faserwerkes,

2. das Vorhandensein von syncytialen Zellen neben "den Deciduazellen" im engeren Sinne,

3. das fast constante Vorkommen von ausserordentlich leicht darstellbaren Centrosomen in den grossen (decidualen) Zellen.

Für die Förderung dieser Untersuchungen bin ich meinem Chef, Herrn Geheimrath v. Winckel, sowie Herrn Professor Dr. Mollier und Herrn Prosector Dr. A. A. Böhm zu grösstem Danke verpflichtet. 
Hörmann, Deciduale Bildungen i. d. Ovarien b. intrauteriner Gravidität. 305

\section{Lit e ratur.}

v. Winckel, Handbuch d. Geburtshülfe. I. 1.

Schmorl, Monatsschr. f. Geb. u. Gyn. Bd. V. H. 1.

Kinoshita, Monatsschr. f. Geb. u. Gyn. Bd. VIII. H. 5.

Schnell, Zeitschr. f. Geb. u. Gyn. Bd. 40. H. 2.

Rabl, Anat. Hefte. Bd. XI. H. 34-37.

Lindenthal, Monatsschr. f. Geb. u. Gyn. Bd. XIII. H. 6.

A. Böhm u. A. Oppel, Taschenbuch der mikroskop. Technik. 1904.

Maresch, K., Centralbl. für allg. Pathologie u. pathol. Anatomie. 16. Bd. No. $16 / 17.1905$.

\section{Erklärung der Abbildungen auf Tafel XI.}

Figur 1. Uebersichtsbild mit deciduaähnlichen Haufen.

Figur 2. Decidualer Haufen, stark vergrössert; Darstellung der intercellulären Fasern.

Figur 3. Zwischen den decidualen Zellen sind Fibroblasten- und Lymphocytenkerne zu sehen.

Figur 4. Syncytiale Bildungen aus den Zellenhaufen.

Figur 5. 6 deciduale Zellen mit Centrosomen und Sphären. 